\title{
Trayectorias de empleabilidad de los jóvenes que abandonan el sistema de protección
}

\section{Employability trajectories of young people leaving protection systems}

\author{
Estefanía Alonso Bello ${ }^{1}$ \\ estefaniaalo@gmail.com \\ Lidia E. Santana Vega \\ Isantana@ull.es \\ Luis Feliciano García \\ Ifelici@ull.edu.es \\ Universidad de la Laguna, España
}

\begin{abstract}
Resumen:
Las trayectorias de empleabilidad de los jóvenes que salen del sistema de protección se ven condicionadas por su situación de exclusión social. Nuestra investigación persigue: a) analizar las trayectorias de los jóvenes extutelados y b) examinar cómo sus experiencias vitales y laborales influyen en su situación de exclusión. Se realizó un estudio de casos múltiples con cuatro jóvenes inmigrantes y tres nacionales. La selección de los casos tuvo en cuenta: a) haber estado acogido al sistema de protección, b) haber pasado un mínimo de 6 meses en un programa de inserción laboral, c) haber transcurrido 6 años después de finalizar el proyecto de inserción. En el estudio se utilizaron entrevistas, planes de inserción, fichas de trabajo, diarios de campo y registros de control para triangular la información y dar credibilidad a los resultados. Los resultados señalan que: 1) Los jóvenes valoran positivamente el apoyo en el proceso de transición al mercado laboral; b) la inserción social y la salida de
\end{abstract}

\begin{abstract}
:
Employability trajectories of young people leaving protection systems are conditioned by their situation of social exclusion. The objectives of this study are: a) to analyse the trajectories of young people leaving public protection systems and b) to analyse how their lives and work experiences affect their social exclusion. A multiple case study was conducted. Four young immigrants and three young nationals participated in the study. The participants were selected on the basis of three criteria: a) they should have lived in residential care institutions, b) they should have been in a job placement program for at least 6 months, c) 6 years should have passed since the end of their job placement program. The data were collected using interviews, insertion plans, worksheets, field notes, control records; this allowed the triangulation of the information and gave the results credibility. The results indicate that: 1) young people value positively the support provided in the transition to the
\end{abstract}

1 Dirección para correspondencia (correspondence address):

Estefanía Alonso Bello. Avd. Trinidad, s/n. Campus Central. 38204, La Laguna. Tenerife (España). 
la situación de exclusión son más factibles con metas claras y un proyecto de vida definido; 3) la escasa formación y experiencia laboral obstaculizan la adquisición y consolidación de competencias de empleabilidad. Los programas de inserción sociolaboral deben fomentar: a) el análisis de los proyectos de vida, b) la ampliación de las redes de apoyo, y c) el diseño de planes de empleo con apoyo.

\section{Palabras clave:}

Competencias de empleabilidad; inserción laboral; exclusión social; jóvenes inmigrantes. labour market; 2) social inclusion and leaving a situation of exclusion are more feasible with clear goals and a well defined life project; 3) poor training and work experience hinder the acquisition and consolidation of employability skills. Labour integration programs should promote: a) the analysis of life projects, b) the expansion of support networks and c) the design of supported employment plans.

\section{Key words:}

Employability competencies; labour insertion; social exclusion; young immigrants.

\section{Résumé:}

Les trajectoires d'employabilité des jeunes qui quittent le système de protection sont conditionnées par leur situation d'exclusion sociale. Notre recherche vise à: a) analyser les trajectoires des jeunes qui étaient sous tutelle, et b) examiner comment leur vie et leurs expériences professionnelles influencent leur situation d'exclusion. Une étude de cas multiple a été menée avec quatre jeunes immigrants et trois ressortissants. La sélection des cas a pris en compte: a) avoir été protégé par le système de protection, b) avoir passé au moins 6 mois dans un programme de placement, c) s'être écoulé 6 ans après la fin du projet d'insertion. Dans cette étude, des entretiens, des plans d'insertion, des feuilles de travail, des journaux de travail et des registres de contrôle ont été utilisés pour trianguler les informations et crédibiliser les résultats. Les résultats indiquent que: 1) les jeunes accordent de l'importance au soutien dans le processus de transition vers le marché du travail; 2) l'insertion sociale et la sortie de la situation d'exclusion sont plus réalisables avec des objectifs clairs et un projet de vie défini; 3 ) Le manque de formation et d'expérience professionnelle entrave l'acquisition et la consolidation des compétences relatives à l'employabilité. Les programmes d'insertion socioprofessionnelle devraient encourager: a) l'analyse des projets de vie, b) l'expansion des réseaux de soutien et c) la conception de plans d'emploi avec soutien.

\section{Mots clés:}

Compétences en employabilité; insertion professionnelle; exclusion sociale; jeunes immigrés.

Fecha de recepción: 7-9-2018

Fecha de aceptación: 2-10-2018 


\section{Antecedentes}

La transición a la vida adulta supone la incursión en el mercado de trabajo (López, Hernández y Viscarret, 1999; Santana Vega, 2013). La inserción laboral es el proceso que permite a las personas mejorar sus condiciones para acceder a un puesto de trabajo. A través del proceso de inserción laboral se incrementan y perfeccionan las competencias de empleabilidad. Estas competencias mejoran la capacidad de obtener/ mantener un empleo y de moverse en el mercado de trabajo, e incluyen habilidades prácticas, actitudes, hábitos, valores sociolaborales, capacidades creativas y recursos psicosociales (Velaz de Medrano, 2005; Organización Internacional del Trabajo, 2015). Las competencias de empleabilidad relativas a la auto-organización, construcción de proyectos académico-laborales, toma de decisiones, resolución de problemas, trabajo en equipo, comunicación, perseverancia, flexibilidad, responsabilidad o corresponsabilidad (Arnau, Marzo, Jariot y Sala, 2013), han de ser aprendidas antes de acceder al mercado de trabajo y perfeccionarse durante la vida laboral (Formichella y London, 2013).

Los jóvenes en riesgo de exclusión social realizan una transición "instantánea" a la vida adulta al salir del sistema de protección que les hace más vulnerables (Avery y Freundichlich, 2009; Geenen y Powers, 2007; Mendes, 2009; Stein, 2006). La exclusión social alude a la falta de acceso a la educación, el empleo, la salud, los sistemas de protección social, el dinero y las relaciones sociales (Laparra, Aguilar y Gaviria, 1996), y se produce por la confluencia de varios procesos de ruptura. Estos procesos ubican a las personas en situación de inferioridad respecto a los recursos, los centros de poder y los valores predominantes (Estivill, 2003; Jiménez, Luengo y Taberner, 2009). La situación psicosocial de jóvenes extutelados se ve agravada por sus características personales: a) baja autoestima, b) escasas habilidades sociales, c) modos de relacionarse con los otros en donde no tiene cabida el diálogo, d) dificultades para aprender conocimientos nuevos, e) miedo e inseguridad, f) rigidez en las ideas sobre sí mismos, g) dependencia excesiva de los adultos, y h) identidad determinada por su condición de extutelados (Anghel y Dima, 2008; Arnau y Gilligan, 2015; Bendit y Hahn, 2008; Dima y Skehill, 2011; Gilligan, 2008; Jariot, Sala y Arnau, 2015; Olmos, 2014; Orteu, 2008).

Varios autores (Courtney y Dworsky, 2006; Davidson, Devaney y Spratt, 2011; Sala, Jariot, Villalba y Rodríguez, 2009; Tilbury, Buys y 
Crees, 2009), señalan que los jóvenes en riesgo de exclusión cuando dejan de ser tutelados se enfrentan a múltiples problemas de salud mental y física y a un apoyo social escaso. Generalmente los jóvenes extutelados no están preparados para cubrir sus necesidades materiales y psicológicas cuando salen del sistema de protección (Arnau et al., 2013). Además tienen una situación administrativa irregular que dificulta su inserción sociolaboral (López Reillo, 2013; Santana, Alonso, y Feliciano, 2016). Dependiendo de la forma en que estos jóvenes afronten la transición al mercado de trabajo, de los recursos/apoyos disponibles y de sus posibilidades/expectativas vitales podrán, o no, salir de la trayectoria de exclusión (Parrilla, Gallego y Moriña, 2010).

La orientación sociolaboral debe ayudarles a: 1) definir objetivos profesionales adecuados, 2) mantener el empleo, y 3) diseñar itinerarios personalizados. Si se quiere ofrecer a los jóvenes extutelados los recursos y las estrategias necesarias para salir de la exclusión, los factores que modulan las trayectorias formativas y profesionales deben ser estudiados por la comunidad científica.

\section{Diseño y metodología}

En este estudio se abordan los siguientes objetivos: a) Analizar las trayectorias de empleabilidad de los jóvenes en riesgo de exclusión una vez salen del sistema de protección. b) Examinar cómo sus experiencias vitales y laborales influyen en su situación de exclusión. Para llevar a cabo la investigación se optó por un diseño de casos múltiples dado que las evidencias obtenidas con este tipo de diseño son más convincentes y el estudio es más robusto (Rodríguez, Gil y García, 1996). Los casos del estudio provienen de la implementación del proyecto de inserción sociolaboral "Subimos juntos la escalera: fomento de la emancipación juvenil" Ilevado a cabo por la Asociación Kanaria de Infancia (Alonso, Santana y Feliciano, 2017). La Tabla 1 recoge las dimensiones de información y los interrogantes específicos de la investigación. 
Tabla 1. Dimensiones de información e interrogantes.

\section{Dimensiones de Interrogantes Información}

\section{Inicio/transcurso del proyecto de inserción}

\begin{tabular}{|c|c|}
\hline $\begin{array}{l}\text { Antecedentes Perso- } \\
\text { nales y Familiares }\end{array}$ & $\begin{array}{l}\text { ¿Por quiénes están constituidas sus familias? } \\
\text { ¿Qué tipo de relación mantienen con sus familias? } \\
\text { ¿Por qué se incorporaron al proyecto? } \\
\text { ¿En cuántos centros han estado? ¿durante cuánto tiem- } \\
\text { po? } \\
\text { ¿En qué situación personal y social se encuentran? }\end{array}$ \\
\hline $\begin{array}{l}\text { Experiencia y Cono- } \\
\text { cimientos Laborales } \\
\text { y Prelaborales }\end{array}$ & $\begin{array}{l}\text { ¿Qué experiencias laborales previas han tenido? } \\
\text { ¿Qué conocimientos tienen de las estrategias de bús- } \\
\text { queda de empleo? }\end{array}$ \\
\hline $\begin{array}{l}\text { Competencias de } \\
\text { Empleabilidad }\end{array}$ & $\begin{array}{l}\text { ¿Qué competencias laborales tienen? } \\
\text { ¿Qué dificultades tienen para acceder al empleo? }\end{array}$ \\
\hline Formación & ¿Qué trayectoria formativa han tenido? \\
\hline Red Social & ¿Son sus redes sociales suficientes y adecuadas? \\
\hline Proyecto de vida & $\begin{array}{l}\text { ¿Cuáles son sus intereses profesionales? } \\
\text { ¿Cuáles son sus expectativas de futuro? }\end{array}$ \\
\hline
\end{tabular}

Antecedentes Perso- ¿En qué situación personal y social se encuentran en la nales y Familiares actualidad? ¿Se han emancipado? ¿Qué relación mantienen con sus familias?

Experiencia y Cono- ¿Cuáles han sido sus trayectorias laborales?

cimientos Laborales ¿Qué puestos han desempeñado y qué duración han tenido sus contratos?

¿Qué le aportó su participación en el proyecto de inserción laboral?

¿Son más autónomos en la búsqueda de empleo?

\begin{tabular}{cl}
$\begin{array}{c}\text { Competencias de } \\
\text { Empleabilidad }\end{array}$ & $\begin{array}{l}\text { ¿Qué factores les han ayudado, o no, a conseguir y } \\
\text { mantener un empleo? }\end{array}$ \\
\hline Formación & $\begin{array}{l}\text { ¿Qué trayectoria formativa han tenido desde que aban- } \\
\text { donaron el proyecto? } \\
\\
\text { ¿Cuáles son los motivos de continuar los estudios o de } \\
\text { abandonarlos? }\end{array}$ \\
\hline Red Social & ¿Qué personas forma su red social de apoyo? \\
& ¿Con quiénes comparten su tiempo?
\end{tabular}

Proyecto de vida ¿Qué metas personales, familiares, formativas y laborales se plantean?

Fuente: elaboración propia. 
A raíz de los planteamientos teóricos nuestras hipótesis de trabajo son:

- Los jóvenes nacionales tienen más dificultades para mantener un empleo que los inmigrantes.

- Los jóvenes nacionales tienen más dificultades para emanciparse que los inmigrantes.

- Los jóvenes nacionales perpetúan la situación de riesgo social frente a los inmigrantes que consiguen salir de ella

- Los jóvenes inmigrantes configuran una red social más amplia que los nacionales.

- Los jóvenes inmigrantes desarrollan más sus competencias de empleabilidad que los nacionales.

- Los jóvenes inmigrantes tienen metas vitales que les llevan a salir de la situación de riesgo, frente a los nacionales que no las tienen.

\section{Participantes}

Nuestro estudio analiza las trayectorias de empleabilidad de siete casos caracterizados por: a) haber estado acogidos al sistema de protección, b) haber participado un mínimo de 6 meses en un proyecto de inserción laboral, y c) haber transcurrido 6 años desde que finalizó el proyecto de inserción. Al inicio de la investigación se realizó una sesión informativa para los jóvenes interesados en participar en el estudio. Posteriormente, se realizó una sesión individual para pactar las claves del proceso con cada joven seleccionado y asegurar su consentimiento informado. Transcurridos 6 años de finalización del proyecto nos pusimos nuevamente en contacto con ellos y se negoció su consentimiento para indagar sobre sus trayectorias de empleabilidad durante ese periodo, asegurándose la confidencialidad del proceso.

\section{Técnicas e Instrumentos}

Las técnicas e instrumentos para la recogida de información se enumeran en la Tabla 2. Las entrevistas iniciales a los jóvenes y a sus tutores aportan información sobre el área familiar, personal, de salud, formativa,... y sobre el nivel de conocimientos prelaborales y laborales. Los planes individuales de inserción recogen la situación de partida de cada joven y sus objetivos. Los diarios de campo dan información sobre las 
sesiones de trabajo con los jóvenes. El informe de prácticas describe el grado de adaptación al puesto de trabajo, las carencias y potencialidades de los jóvenes, etc. Los informes de baja reflejan la evolución de cada joven en el proyecto: los objetivos conseguidos, los que debe conseguir y por qué motivo causa baja. Las entrevistas finales ofrecen información sobre lo que el proyecto aportó a los jóvenes, el desarrollo de sus competencias de empleabilidad, sus trayectorias laborales y su situación personal y familiar.

Tabla 2. Dimensiones de información, técnicas e instrumentos.

\begin{tabular}{|c|c|c|c|c|c|c|c|}
\hline \multirow[t]{2}{*}{ Dimensiones } & \multicolumn{7}{|c|}{ Técnicas e instrumentos } \\
\hline & EIJ & ET & PII & DC & IP & IB & EFJ \\
\hline Situación Personal y Familiar & $\mathrm{x}$ & $\mathrm{x}$ & $\mathrm{x}$ & $\mathrm{x}$ & $\mathrm{x}$ & $\mathrm{x}$ & $\mathrm{x}$ \\
\hline Experiencia/Conocimientos Laborales & $x$ & $\mathrm{x}$ & $\mathrm{x}$ & & & & $\mathrm{x}$ \\
\hline Competencias de Empleabilidad & $x$ & $\mathrm{x}$ & $\mathrm{x}$ & $\mathrm{x}$ & $\mathrm{x}$ & $\mathrm{x}$ & $\mathrm{x}$ \\
\hline Formación & $x$ & $x$ & $x$ & $x$ & & $x$ & $x$ \\
\hline Red Social & $\mathrm{x}$ & $\mathrm{x}$ & $\mathrm{x}$ & $\mathrm{x}$ & & $\mathrm{x}$ & $\mathrm{x}$ \\
\hline Proyecto de vida & $\mathrm{x}$ & $\mathrm{x}$ & $\mathrm{x}$ & $\mathrm{x}$ & & $\mathrm{x}$ & $\mathrm{x}$ \\
\hline
\end{tabular}

EIJ: Entrevista Inicial a los Jóvenes; ET: Entrevista a Tutores; PPI: Planes Individuales de Inserción; DC: Diario de Campo; IP: Informes de Prácticas; IB: Informes de Baja; EFJ: Entrevista Final a los Jóvenes.

\section{Procedimiento}

La investigación se desarrolló en tres fases: 1) La primera describía los procesos de inserción sociolaboral de cada joven durante su participación en el proyecto, y supuso el trabajo directo e individual con los participantes; esta fase culminó con la redacción de los informes de los siete casos analizados. 2) En la segunda se realizó un estudio comparativo de los siete casos, analizando los aspectos comunes y diferenciales del proceso de inserción sociolaboral de los jóvenes. 3) En la tercera se analizó sus trayectorias de empleo y los factores que las modulaban seis años después de finalizar el proyecto de inserción sociolaboral.

\section{Análisis de Datos}

El análisis de la información obtenida en las entrevistas, los planes individuales de inserción, las fichas de trabajo, los diarios de campo y los informes de prácticas y de baja se llevó a cabo mediante el análisis de 
contenido cualitativo. A través del mismo se realizó la reducción de la información a unidades de contenido significativo, mediante un proceso de selección, focalización, simplificación y abstracción de los datos. Con el fin de asegurar la credibilidad de los datos se puso en práctica una serie de estrategias: a) la triangulación de fuentes y técnicas, b) la comprobación con participantes, c) la recogida abundante de datos.

\section{Resultados y discusión}

En este apartado se describe cada caso destacando los factores que han modulado sus trayectorias vitales después de que salieran del sistema de protección.

Caso 1. Ancor fue declarado en desamparo con 9 años junto a sus tres hermanos con los que vivió en tres centros de acogida. Los contactos con la familia biológica eran muy escasos. A los 18 años residió en un piso tutelado. Estudió hasta $2^{\circ}$ de la ESO y su nivel en lectoescritura y expresión oral era elemental. Sabía cómo buscar empleo y tenía experiencia laboral, pero era muy dependiente de la figura del adulto. Su red social la formaban personas con las que había convivido en el centro de acogida. Abandonó el proyecto porque consiguió un empleo por un periodo de dos meses prorrogables. La situación de Ancor seis años después es la siguiente:

Contexto familiar y red social. Ancor tiene actualmente 24 años. Está viviendo con su pareja en la casa de su suegra. Tienen un hijo de dos años y se relaciona bien con sus padres. Su tiempo libre lo comparte con la familia y con algunos excompañeros de trabajo.

Formación y trayectoria laboral. Su trayectoria laboral se reduce a dos años repartidos en cuatro empresas diferentes. Actualmente está en paro, busca activamente trabajo, cobra una prestación que se le acaba, y debe pedir la ayuda familiar. Según él su situación de desempleo obedece a factores como: poca experiencia en otros trabajos, no disponer de carné de conducir y sus escasos estudios. Está sacando el carné de conducir y su idea es sacarse la ESO cuando disponga de tiempo ya que debe ocuparse de su hijo, mientras su pareja trabaja.

Proyecto de vida. Sus metas de futuro son tener una vida con su familia, tener un coche, conseguir una casa y realizar un curso ocupacional. Valoración del proyecto de inserción laboral. Valora de forma muy 
positiva todos los aspectos del proyecto: los conocimientos impartidos, los recursos, el personal, y el apoyo recibido en momentos trascendentes de la vida.

Caso 2. Marcos fue declarado en desamparo a los 5 años. Cursó $2^{\circ}$ de la ESO y realizó un Programa de Cualificación Profesional. Comenzó el proyecto con 16 años, pero sin continuidad. A los 17 años retomó su participación en el proyecto, ampliando sus conocimientos sobre la búsqueda de empleo; poseía experiencia laboral que no amplió porque abandonó sus prácticas. No sabía gestionar los conflictos, le faltaba autonomía y tenía dificultad para expresarse oralmente. Se relacionaba con otros jóvenes en su misma situación y casi no tenía contacto con su familia. Quería ser cocinero y abandonó el proyecto cuando comenzó a participar en un taller de cocina. La situación de Marcos seis años después es la siguiente:

Contexto familiar y red social. Tiene 22 años y vive con su pareja. La relación con sus padres es regular y buena con sus hermanas. Se relaciona principalmente con su pareja, su suegra, su hermano mayor y su sobrino.

Formación y trayectoria laboral. Dejó la escuela taller para formarse como cocinero porque surgieron problemas que no supo resolver. Estuvo trabajando como peón agrícola durante seis meses, pero una vez que finalizó este empleo no surgieron nuevas ofertas. Posee conocimientos para buscar empleo, aunque tendría que renovarlos, y manifiesta seguir teniendo dificultades para expresarse. En su opinión los motivos para estar desempleado son: no tener estudios, no tener carné de conducir y falta de experiencia laboral. Ha abandonado el mercado laboral y tiene una pensión no contributiva por un diagnóstico de fatiga muscular

Proyecto de vida. Aunque la pensión apenas le da para pagar el alquiler, sus sueños no están relacionados con obtener un futuro mejor, sino con casarse algún día e irse de viaje de novios.

Caso 3. Mateo fue declarado en desamparo a los 7 años y estuvo en dos centros de acogida. Estudió hasta $2^{\circ}$ de la ESO e inició un Programa de Garantía Social, ambos sin finalizar. Empezó a participar en el proyecto con 16 años. Poseía algunos conocimientos pre-laborales y no tenía experiencia laboral. Durante el proyecto amplió estos conocimientos y adquirió más autonomía pero no obtuvo experiencia laboral al abandonar las prácticas. Mateo era poco responsable, dependiente, con escasa motivación y bastante tímido. Su red social estaba formada por 
su familia y los jóvenes de su centro. No tenía claro su proyecto de vida. Abandonó el proyecto por continuas faltas de asistencia. La situación de Mateo seis años después es la siguiente:

Contexto familiar y red social. Vive con su madre y su hermana. Su padre murió. Manifiesta tener una relación fantástica con su madre y sus cuatro hermanos mayores, sus tíos y abuela materna. Su tiempo libre lo comparte con su novia, las amigas de ésta y sus amigos.

Formación y trayectoria laboral. Acabó $3^{\circ}$ de la ESO y está haciendo un curso ocupacional. Estuvo trabajando como limpiador durante 6 meses y en la actualidad está desempleado. No recibe ningún ingreso, pero su madre y su novia le ayudan económicamente. Cree que el motivo por el que no consigue empleo es porque necesita más experiencia para estar mejor preparado.

Proyecto de vida. Irse a vivir con su novia que vive emancipada en la casa de su abuela.

Valoración del proyecto de inserción laboral. Mateo valora los aspectos del proyecto de inserción positivamente; considera que ha sido un recurso muy útil porque ha aprendido muchas cosas, tales como saber buscar un empleo, enfrentarse a una entrevista, etc.

Caso 4. Abdou llegó a España con 17 años, vivió en un macrocentro hasta cumplir la mayoría de edad, luego residió en un piso tutelado. No mantenía contacto con su familia al desconocer su paradero. Se preocupaba por ampliar su formación. Cuando abandonó el proyecto, cursaba $3^{\circ}$ de la ESO, $4^{\circ}$ de español y $6^{\circ}$ de inglés. En el proyecto de inserción aprendió estrategias de búsqueda de empleo, así como conocimientos y destrezas laborales; en el plano personal consiguió una mayor autonomía y estrategias para tolerar la frustración y promover el autodesarrollo personal. Tenía experiencia laboral previa en su país, que se incrementó en el país de acogida con trabajos temporales y prácticas en empresas. Contaba con amigos/as de diferentes nacionalidades y se sentía integrado. Era puntual, responsable, cuidaba sus materiales, y participaba en las acciones formativas. Consiguió un contrato durante un año y regularizó su situación administrativa. Se fue a vivir con una familia, pero su deseo era independizarse. Una vez consolidada su inserción laboral, su idea era seguir formándose y compaginar el trabajo con los estudios. La situación de Abdou es, seis años después, la siguiente:

Contexto familiar y red social. Abdou se ha independizado y comparte piso con otros jóvenes; ha localizado a su madre y hermanos en 
Senegal y ha podido visitarlos. Su tiempo libre lo comparte con amigos y compañeros, nacionales y extranjeros. Se siente muy integrado.

Formación y trayectoria laboral. Ha dejado los estudios por incompatibilidad de horarios con su trabajo, pero no descarta la idea de retomarlos porque es consciente de su importancia para optar a otras alternativas. Tras dejar su primer empleo, comenzó a trabajar en una empresa de reciclaje; tiene un contrato de trabajo indefinido en una fábrica en la que lleva tres años.

Proyecto vital. Desea finalizar sus estudios, sacarse el carné de conducir y marcharse a vivir a los Estados Unidos. Sin embargo este paso lo dará siempre y cuando lo vea claro, de lo contrario se quedará en Tenerife.

Valoración del proyecto de inserción laboral. Abdou valora de forma positiva los recursos y los conocimientos adquiridos en el proyecto, pero echa en falta una formación para la vida, ya que solo se fijaban objetivos a corto plazo. Valora negativamente las prácticas porque considera que al empresariado sólo le interesa tener a personas que trabajen de forma gratuita, sin preocuparse realmente por enseñar bien un oficio.

Caso 5. Moussa llegó a España con 16 años, residió en un macrocentro y al cumplir 18 pasó a vivir en un piso tutelado. Su familia residía en África, excepto un hermano mayor que vivía en la península y su padre en Francia. Mantenía contacto telefónico frecuente con ellos. Tenía experiencia laboral en África. Durante el proyecto realizó prácticas, pero no le abrieron las puertas al mercado laboral porque el empresario consideró que no tenía el perfil adecuado para el puesto de trabajo. Adquirió conocimientos y autonomía para la búsqueda de empleo. Se mostraba predispuesto, dinámico y motivado para conseguir empleo. Fue puntual, asistía con asiduidad, cumplía con la normativa y participaba en todas las acciones. Cuando abandonó el proyecto no había conseguido el permiso de trabajo. Realizó varios cursos de formación ocupacional y se matriculó en una autoescuela. Poseía conocimientos de inglés, francés y árabe. Su red social se amplió, encontró pareja y se fue a vivir con ella. Su meta era conseguir un empleo y regularizar su situación para aumentar sus propias posibilidades y las de su familia. El intento por conseguir los papeles a toda costa le llevó a no adquirir una correcta cultura de trabajo y cometer errores (mentir en el curriculum vitae, tener dificultades para acatar las normas o instrucciones del puesto de trabajo, tener demasiada prisa, trabajar de forma ilegal ante la promesa de con- 
tratos, ...) que jugaron en su contra. La situación de Moussa es, seis años después, la siguiente:

Contexto familiar y red social. Moussa vive con su hermano menor en Dinamarca, se casó y tiene una niña; ellas viven en Senegal. Su padre está en Francia y el resto de su familia está en Senegal. Mantiene contacto con todos y cada cierto tiempo se va a Senegal.

Formación y trayectoria laboral. Está centrado en trabajar y ganar dinero y no tiene tiempo de estudiar. Antes de salir de España sacó el carné de conducir, lo que le ayudó a conseguir el empleo actual y cuando llegó a Dinamarca hizo un curso obligatorio de idioma. Ha viajado por muchos países de Europa en busca de su objetivo. Estuvo en Francia, de allí fue a Suiza. Marchó a Suecia donde permaneció un año trabajando ilegalmente. Más tarde estuvo en Noruega donde tuvo que dormir en la calle. Finalmente llegó a Dinamarca y, por medio de un amigo suyo, consiguió empleo y regularizó su situación en el país. Tuvo que pasar antes por un periodo de prácticas, lo superó y lleva trabajando durante dos años en una empresa.

Proyecto vital. Su ilusión es crear una granja de pollos con su hermano en Senegal para venderlos congelados. Cuando va a Senegal compra cosas y así va creando poco a poco su empresa.

Valoración del proyecto de inserción laboral. Cuando llegó a España, había muchas cosas que desconocía y aprendió en el proyecto de inserción. El aprendizaje adquirido le ha servido y lo ha utilizado a lo largo de su vida. Todos los aspectos del proyecto los valora muy positivamente. Crítica la política del Gobierno español que lo mantuvo y le dio formación hasta los 18 años y luego le impidió trabajar a causa de "los papeles". Ha conseguido su objetivo gracias a su fuerza de voluntad, a lo que aprendió en el proyecto de inserción laboral y a pensar de forma positiva; "siempre se pueden alcanzar los sueños, basta con proponérselo y trabajar duro".

Caso 6. Ousmane llegó a España en el 2000. Estuvo dos años tutelado. Se incorporó al proyecto con 18 porque buscaba trabajo de manera infructuosa. Sus preferencias profesionales no estaban definidas, su objetivo era trabajar y ayudar a su familia. Estaba residiendo en un piso tutelado para jóvenes inmigrantes mayores de edad. Sus padres y sus siete hermanos estaban en África y mantenía contacto con ellos. Su lengua materna era el francés. Hizo un curso de inglés básico y varios de formación ocupacional. Para él la formación era importante y no descartaba la 
idea de seguir estudiando. Fue puntual, responsable, entusiasta y estaba muy motivado por aprender y conseguir sus metas. Había estado inscrito en otros proyectos de inserción sociolaboral pero desconocía el uso de las nuevas tecnologías y de otros medios de búsqueda de empleo. No poseía permiso de residencia ni de trabajo y tenía dificultades con el idioma. Su red social se fue ampliando gracias a su participación en varias acciones formativas, en las prácticas en empresas y en iniciativas juveniles de ocio. Tenía experiencia laboral; al finalizar las prácticas en empresa fue contratado y pudo regularizar su situación administrativa y se emancipó. La situación de Ousmane es, seis años después, la siguiente:

Contexto familiar y red social. Vive en un piso compartido con amigos. Fue a Mali a casarse pero vuelve solo a Tenerife; mantiene contacto frecuente con su familia.

Formación y trayectoria laboral. No está estudiando porque no tiene ni tiempo, ni ganas, debido a su trabajo. Dispone de carné de conducir y tiene coche. Abandonó su primer empleo y trabajó, previo periodo de prácticas, en una empresa durante dos años. En la actualidad tiene un trabajo fijo en una finca como peón de agricultura y empaquetado. En su opinión, lo que le ha ayudado a conseguir los empleos ha sido su forma de hablar, su presencia, sus ganas de trabajar, tener el carné de conducir y tener sus papeles en regla, ya que dispone de la tarjeta permanente.

Proyecto vital. Quiere volver a África y montar un negocio de electrodomésticos y materiales de construcción. Ahora intenta ahorrar para poder ir dando forma a su futura empresa.

Valoración del proyecto de inserción laboral. Considera que es de utilidad para los jóvenes, sobre todo para los inmigrantes. Valora muy positivamente todos los aspectos del proyecto, salvo las prácticas realizadas en las empresas, que deben contemplar menos horas.

Caso 7. Modou llegó a Tenerife en 2006 con 14 años y estuvo en un centro de protección hasta los 18 años, después residió en un piso tutelado. Una de sus metas al cumplir la mayoría de edad era marcharse a Barcelona y vivir con su hermano que trabaja en la construcción, pero no pudo cumplirla. Modou rehusaba hablar de su familia, que vivía en Mali y con la que mantenía contacto frecuente. Comenzó a participar en el proyecto de inserción con 16 años; pero solo durante dos meses porque se le inscribió en un Programa de Cualificación Profesional Inicial. Con 18 años reinició el proyecto motivado por su tutor para fomentar su autonomía y adquirir estrategias de búsqueda de empleo. El joven 
no poseía experiencia laboral ni había buscado empleo. Tenía estudios sin finalizar hasta $3^{\circ}$ de la ESO y un curso de formación ocupacional. Acudía con regularidad al proyecto y era puntual, pero su participación estuvo caracterizada por la desmotivación, la desgana y las dificultades con el idioma; además, presentaba escasas habilidades sociales y de resolución de conflictos, una baja tolerancia a la frustración, y adoptaba actitudes infantiles. Su principal dificultad era entender y hacerse entender, y sus problemas con la lectoescritura obstaculizan su aprendizaje. Paulatinamente fue aumentando su red social por medio de su participación en talleres, y en actividades organizadas por iniciativas juveniles. Gracias a las prácticas en empresas, Modou consiguió un contrato en una lavandería por un año y pudo regularizar su situación administrativa. La situación de Modou es, seis años después, la siguiente:

Contexto familiar y red social. Comparte piso con amigos y mantiene contacto con su familia, aunque no es regular. En pocos meses se irá a Mali para casarse.

Formación y trayectoria laboral. En su opinión es bueno saber cosas pero ahora no está estudiando. Abandonó su primer empleo y se fue un año a la península con su hermano donde trabajó un mes como peón; volvió a Tenerife y le contrataron en su anterior empresa de manera indefinida.

Proyecto vital. Quiere volver a África y hacerse una casa y quedarse a vivir allí. No se plantea el tema del trabajo porque según manifiesta en su país no es necesario tener un trabajo para vivir, "cultivas tu comida y listo". Por el momento trabaja para ahorrar y hacerse la casa.

Valoración del proyecto de inserción laboral. El proyecto de inserción le ha permitido conseguir trabajo, madurar y aprender muchas cosas; gracias a él tiene contrato indefinido y residencia. Valora todos los aspectos del proyecto, pero mejoraría la enseñanza del idioma para hablar y escribir bien en español.

\section{Conclusiones}

En nuestra investigación partimos de un significado amplio de exclusión social, entendida como distanciamiento del individuo de la sociedad y su incapacidad para participar efectivamente en la vida económica, social y cultural de la comunidad (Fangen, 2012; Paolini, 2013). 
Los resultados del estudio muestran que los jóvenes nacionales extutelados están en la actualidad desempleados, sus recursos económicos son escasos y viven con familiares, mientras que los jóvenes inmigrantes extutelados se encuentran trabajando por cuenta ajena y se han emancipado. Los jóvenes inmigrantes al incorporarse al mercado laboral han salido de la situación de exclusión y se han insertado en la sociedad. La situación de desempleo de los jóvenes nacionales limita su participación en la sociedad y perpetúa su trayectoria de exclusión.

La red social de apoyo constituye una dimensión muy importante para los jóvenes extutelados. Los jóvenes inmigrantes han ampliado su red social de una manera mucho más notoria que los jóvenes nacionales, quienes, en algunos casos, incluso la han reducido a sus círculos más íntimos. La no ampliación o reducción de esta red tiene consecuencias negativas para los jóvenes extutelados. Según Paolini (2013) si las redes de apoyo son restringidas, la probabilidad de distanciamiento de la sociedad es mayor, disminuyendo las posibilidades de participar en la comunidad. Los proyectos de inserción deben trabajar transversalmente la ampliación de la red social de apoyo como estrategia para salir de las situaciones de exclusión, de obtener y mantener un empleo, y de moverse en el mercado laboral (Velaz de Medrano, 2005).

Los jóvenes nacionales siguen necesitando desarrollar sus competencias de empleabilidad. Su falta de formación y sus escasas experiencias laborales son un obstáculo para la adquisición y la consolidación de dichas competencias. Por el contrario, la experiencia laboral acumulada por los jóvenes inmigrantes les ha ayudado a desarrollar y mejorar sus competencias de empleabilidad tales como: la capacidad de aprender y cumplir con las expectativas del empleador, la paciencia, la capacidad de interactuar de forma positiva con los clientes y compañeros de trabajo, y la capacidad para solucionar conflictos de forma positiva (Arnau y Gilligan, 2015). Según Formichella y London (2013) es necesario aprender las competencias de empleabilidad a una edad temprana y perfeccionarlas durante la trayectoria laboral. En los jóvenes nacionales extutelados, sus limitadas trayectorias en el mercado de trabajo dificultan el desarrollo de las competencias de empleabilidad, y condicionan su proceso de inserción laboral. El trabajo y la experiencia es un importante medio para que los jóvenes extutelados puedan salir de la situación de exclusión (Arnau y Gilligan, 2015).

Desde su llegada al país de acogida los jóvenes inmigrantes tienen 
definidos sus objetivos personales y laborales a corto y medio plazo. La claridad de sus metas se mantiene a lo largo de su periplo vital tras abandonar el sistema de protección. Esta circunstancia contribuye al empeño que dichos jóvenes ponen en el desarrollo de su proyecto de vida. Sin embargo, los jóvenes nacionales muestran una mayor indefinición en sus metas vitales, generalmente planteadas a muy corto plazo, y un proyecto de vida poco estructurado. A la falta de concreción de dicho proyecto vital se suma la dependencia excesiva del mundo adulto y las redes debilitadas de apoyo, lo que obstaculiza los procesos de inserción y emancipación (Dima y Skehill, 2011; Olmos, 2014). En las sociedades regidas por la modernidad líquida (Bauman, 2000) las trayectorias vitales y profesionales son cada vez más impredecibles. Las notas características del desarrollo de dichas trayectorias son la flexibilidad, la adaptabilidad, la formación continua y la toma de decisiones a lo largo de la vida. Sin embargo, esa flexibilidad no implica indefinición del proyecto personal de vida. Los jóvenes extutelados, al igual que los jóvenes de su edad, necesitan clarificar sus metas profesionales, académicas y familiares, y tener la suficiente capacidad de adaptación para ajustarlas a los cambios que el contexto socioeconómico imprime a sus trayectorias. Esta capacidad de adaptación está vinculada al autodesarrollo del individuo, quien debe asumir el compromiso de planificar su vida profesional y tener una actitud proactiva a la hora de buscar oportunidades.

Los jóvenes extutelados consideran importante y valoran positivamente la atención/ apoyo en el proceso de transición al mundo del trabajo. En el actual periodo de crisis los jóvenes tardan más tiempo en emanciparse; por tanto aprecian sobremanera los recursos estables y específicos ofrecidos por el proyecto de inserción laboral. Pero mientras tales recursos facilitaron la transición al empleo de los jóvenes inmigrantes, no ocurrió lo mismo con los jóvenes nacionales; para ellos sería necesario desarrollar estrategias alternativas que: 1) compensen sus carencias, 2) les permita analizar sus proyectos de vida, 3) les forme para la inserción laboral, y 4) les oriente a través de planes de empleo con apoyo (Arnau y Gilligan, 2015). Los jóvenes nacionales son derivados a edad temprana a centros de protección, con la finalidad de dar respuesta inmediata a su situación sociofamiliar. La derivación a centros de protección es una solución cómoda para las instituciones responsables de los menores pero no resuelve de manera efectiva todas sus necesidades (Tilbury et al. 2009).

Los resultados del estudio deben ser considerados a la luz de varias 
limitaciones. Es necesario disponer de un mayor número de sujetos que nos permita corroborar las tendencias observadas en los jóvenes nacionales e inmigrantes en riesgo de exclusión. El estudio se realizó solo con varones que estaban implicados en un programa de inserción laboral, lo cual plantea una duda razonable sobre si las conclusiones obtenidas serían parecidas en el caso de que los sujetos sean mujeres y/o no hayan participado en este tipo de proyectos.

El estudio abre nuevas vías para la investigación sobre los procesos de transición y emancipación de estos jóvenes. Cabe plantearse las siguientes cuestiones para seguir investigando: ¿Qué diferencias y similitudes tienen los chicos y chicas extutelados en sus trayectorias laborales? ¿Qué estrategias inserción laboral facilitan su incorporación al empleo y el mantenimiento del puesto de trabajo? ¿Qué diferencias presentan los jóvenes en riesgo de exclusión respecto a la inteligencia emocional, las competencias de empleabilidad y los proyectos de vida según el sexo, edad y origen? ¿Cómo el sistema de protección ha de afrontar los procesos de inserción para que los jóvenes obtengan un empleo, y sepan moverse en el mercado laboral? ¿De qué manera los planes de empleo con apoyo facilitan su inserción laboral? Comprender los procesos por los que los jóvenes salen o no de las trayectorias de exclusión y ofrecer líneas de intervención para su transición a la vida adulta constituye un prometedor campo para futuras investigaciones, y para diseñar políticas que den una respuesta educativa a los jóvenes en riesgo de exclusión social.

\section{Referencias}

Anghel, R. y Dima, G. (2008). Romania. En M. Stein, \& E. Munro (Eds.), Young people's transitions from care to adulthood. International research and practice. London: Jessica Kingsley.

Arnau, L., Marzo, M., Jariot, M. y Sala, J. (2013). Learning basic employability competence: a challenge for the active labour insertion of adolescents in residential care in their transition to adulthood. European Journal of Social Work, 17 (2), 252-265.doi:1 $0.1080 / 13691457.2013 .802227$

Arnau, L. y Gilligan, R. (2015). What helps young care leavers to enter the world of work? Possible lessons learned from an exploratory study in Ireland and Catalonia. Children and Youth Services Review, 53, 185-191.doi:10.1016/j.childyouth.2015.03.027

Avery, R. y Freundich, M. (2009). You're all grown up: termination of foster care sup- 
Trayectorias de empleabilidad de los jóvenes que abandonan el sistema de protección Estefanía Alonso Bello, lidia E. Santana Vega y luis Feliciano García

port at age 18, Journal of Adolescence, 32 (2), 247-257. doi: 10.1016/j.adolescence .2008.03. 009

Bauman, Z. (2000). Liquid Modernity. Cambridge: Polity Press.

Courtney, M. y Dworsky, A. (2006). Early outcomes for young adults transitioning from out-of-home care in the USA. Child and Family Social Work, 11, 209-219. doi: 10.1111/j.1365-2206.2006.00433.x

Bendit, R. y Hahn, M. (2008). Youth transitions: processes of social inclusion and patterns of vulnerability in a globalized world. Budrich: Leverkusen-Opladen.

Davidson, G., Devaney, J. y Spratt, T. (2011). The impact of adversity in childhood on outcomes in adulthood. Journal of Social Work, 10 (49), 369390. doi:10.1177/1468017310378783

Dima, G. y Skehill, C. (2011). Making sense of leaving care: The contribution of Bridges model of transition to understanding the psycho-social process. Children and Youth

Services Review, 33(12), 2532-2539.

Estivill, J. (2003). Concepts and strategies for combating social exclusion. Ginebra: International Labour Office.

Fangen, K. (2012). Social Exclusion and Inclusion of Young Immigrants in Different Arenas. Outline of an Analytical Framework. En A. Lopez-Varela (Ed.), Social Sciences and Cultural Studies - Issues of Language, Public Opinion, Education and Welfare, (pp. 265-278). Rijeka: InTech

Formichella, M. y London, S. (2013). Empleabilidad, educación y equidad social. Revista de Estudios Sociales, 47, 79-91.

Gilligan, R. (2008). Promoting resilience in young people in long-term care. The relevance of roles and relationships in the domains of recreation and work. Journal of Social Work Practice, 22(1), 37-50.

Greenen, S. y Powers, L. (2007). Tomorrow is another problem: The experiences of youth in foster care during their transition into adulthood, Children and Youth Services Review, 29, 1085-1101. doi: 10.1016/j.childyouth.2007.04.008

Jiménez, M., Luengo, J. y Taberner, J. (2009). Exclusión social y exclusión educativa como fracasos. Concepto y líneas para su compresión e investigación. Profesorado. Revista de currículum y formación del profesorado, 13 (3), 11-49.

Jariot, M., Sala, J. y Arnau, L. (2015). Jóvenes tutelados y transición a la vida independiente: indicadores de éxito. Revista Española de Orientación y Psicopedagogía, 26 (2), 90-103.

Laparra, M., Aguilar, M. y Gaviria, M. (1996). Inserción por la actividad económica: un nuevo horizonte para los servicios sociales. En M.C. Alemán y J. Garcés (Comp), Administración social: servicios de bienestar social (pp.633-678) Madrid: Siglo XXI.

López Reillo, P. (2013). Young African migrants reinventing their lives in the Canary Islands. Shima. The International Journal of Research into Island Culture, 7 (2), 39-54.

López, A., Hernández, J. y Viscarret, J. (1999). Jóvenes en una sociedad segmentada. Valencia: Nau Llibres.

Mendes, P. (2009). Young people transitioning from state out-of-home care. Jumping hoops to access employment. Australian Institute of Family Studies, 83, 32-38. doi:10.1111/j.1365-2206.2010.00749x 
Olmos, P. (2014). Competencias básicas y procesos perceptivos: Factores y claves en la formación y orientación de los jóvenes en riesgo de exclusión educativa y sociolaboral. Revista de Investigación Educativa, 32 (2), 531-546. doi: 10.6018/rie.32.2.181551

Organización Internacional del Trabajo (2015). Competencias para el empleo. Mejorar la empleabilidad de los jóvenes: La importancia de las competencias clave. Recuperado en http://www.ilo.org/skills/pubs/WCMS_371815/lang--es/index.htm

Orteu, X. (2008). La inserción como modelo de intervención. IV Congreso Multidisciplinar Trastornos del Comportamiento en Menores. Terapias aplicadas en la escuela, la familia, la salud y los sistemas de protección y justicia. Palma de Mallorca, 07-06 de marzo.

Paolini, G. (2013). Youth Social Exclusion and Lessons from Youth Work. Bruselas: EACEA/Comisión Europea. Recuperado de http://eacea.ec.europa.eu/youth/tools/documents/social_exclusion_and_youth_work.

Parrilla, A., Gallego, C. y Moriña, A. (2010) El complicado tránsito a la vida activa de jóvenes en riesgo de exclusión: una perspectiva biográfica. Revista de Educación, $351,211-233$.

Rodríguez, G., Gil, J. y García, E. (1996). Metodología de la investigación cualitativa. Málaga: Aljibe.

Sala, J., Jariot, M., Villalba, A. y Rodríguez, M. (2009). Analysis of factors involved in the social inclusion process of young people fostered in residential care institutions. Children and Youth Services Review, 31 (12), 1251-1257. doi: 10.1016/j.childyouth.2009.05.010 .

Alonso Bello, E.; Santana Vega, L.E. y Feliciano García, L. (2017). Proyecto de inserción laboral. ¿Subimos juntos la escalera? Revista Electrónica de Investigación y Docencia (REID), 18, 69-82. DOI: https://dx.doi.org/10.17561/reid.v0i18.3331.

Santana Vega, L.E., Alonso Bello, E., y Feliciano García, L. (2016). La inserción sociolaboral de jóvenes en riesgo de exclusión social. Revista Española de Orientación y Psicopedagogía, 27(3), 61-75. doi: http://dx.doi.org/10.5944/reop.vol.27. num.3.2016.18800.

Santana Vega, L.E. (2013). Orientación profesional. Madrid: Síntesis.

Stein, M. (2006). Research review: Young people leaving care. Child and Family Social Work, 11 (3), 273-279. doi: 10.1111/j.1365-2206.2006.00439.x

Tilbury, C., Buys, N. y Creed, P. (2009). Perspectives of young people in care about their scool-to-work transition. Australian Social Work, 62, 476-490. doi: 10.1080/03124070903312849.

Velaz de Medrano, C. (2005). Medidas para prevenir el rechazo escolar y evitar la exclusión social desde un enfoque democrático y comunitario de la atención a la diversidad. En VV.AA., Educación para la ciudadanía (pp. 90-108). Madrid: Atlántida. 
